\title{
Weakening of the repressive $Y Y-1$ site on the thrombospondin-1 promoter via C-Jun/YY-1 interaction
}

\author{
Jung-Hoon Kang ${ }^{1}$, Seo-Yoon Chang ${ }^{1}$, \\ Dong-Hoon Yeom ${ }^{1}$, Soo-A Kim ${ }^{2}$, \\ Soo-Jong $\mathrm{Um}^{3}$ and Kyong-Ja Hong ${ }^{1,4}$ \\ ${ }^{1}$ Department of Biochemistry \\ College of Medicine, The Catholic University of Korea \\ 505 Banpo-dong, Socho-gu \\ Seoul 137-701, Korea \\ ${ }^{2}$ Department of Pathology \\ College of Medicine \\ The Chosun University of Korea \\ Seoseok-dong, Dong-gu \\ Gwangju 501-759, Korea \\ ${ }^{3}$ Department of Bioscience and Biotechnology \\ Sejong University, 98 Gunja-dong \\ Gwangjin-gu, Seoul 143-747, Korea \\ ${ }^{4}$ Corresponding author: Tel, 82-2-590-1176; \\ Fax, 82-2-596-4435; E-mail, kjhong@catholic.ac.kr
}

Accepted 28 June 2004

Abbreviations: CMF-PBS, calcium/magnesium-free phosphate-buffered saline; EMSA, electrophoretic mobility shift assay; GAPDH, glyceraldehyde-3 phosphate dehydrogenase; PCR, polymerase chain reaction; PMA, phorbol 12-myristate 13-acetate; nt(s), nucleotide(s); TSP-1, thrombospondin-1; YY-1, Yin Yang-1

\section{Abstract}

Thrombospondin-1 (TSP-1) level is tightly regulated at the transcriptional level. To determine the detailed molecular mechanisms of TSP-1 expression, nine serial 5 '-deletion constructs of the human genomic tsp-1 promoter (nucleotides $-2,220$ to +756 ) were prepared, inserted into luciferase reporter plasmids, and transiently transfected into the Hep3B human hepatocarcinoma cell. Among the nine 5'-deletion constructs, PTSP-Luc-4 $(-767 \sim+756)$ had consistently decreased luciferase activity with or without PMA stimulation, whereas a further truncated construct [pTSP-Luc-4' $(-407 \sim+756)$ ] had increased levels of expression. By searching the nucleotides from -767 to -407 , a consensus binding sequence (5'-CCATTTT-3') for the repressor Yin Yang-1 (YY-1) at nucleotide -440 was identified. The suppression induced by this site was weakened in the presence of the region upstream of nucleotide -767 (pTSP-Luc-1 and -2). Nuclear protein directly bound to an oligonucleotide containing the repressive YY-1 sequence but the binding capacity of the sequence was decreased by the increased c-Jun levels. Moreover, proteins immunoprecipitated with anti-YY-1 revealed an interaction between C-Jun and YY-1 factor. These data suggest that the repressive YY-1 site of the tsp-1 promoter could not be functional via activating positive cis-elements on the upstream from this site and weakened via c-Jun/YY-1 interactions.

Keywords: c-Jun; gene expression; promoter; TSP-1; YY-1

\section{Introduction}

Thrombospondin-1 (TSP-1) is a homotrimer in which each subunit contains multiple domains. TSP-1 is thus involved in a number of biological processes, including cell growth, migration, and adhesion (Lawler, 1986; Bornstein, 1992; Laharv et al., 1993; Robert et al., 1996; Chen et al., 2000). Although the effects of TSP-1 on tumorigenesis and angiogenesis are controversial, an increasing evidence has shown that TSP-1 acts as a negative regulator in solid tumor progression and neovascularization (Weinstat et al., 1994; Iruela-Arispe et al., 1999; Streit et al., 1999; Jimenez et al., 2000; Nor et al., 2000). For example, transfer of TSP-1 CDNA into a human breast carcinoma cell line suppresses primary tumor proliferation, metastatic potential, and angiogenesis (Nor et al., 2000).

Previous reports have documented that TSP-1 is synthesized by numerous cells and is widely distributed throughout all tissues (Mosher et al., 1982; Jaffe et al., 1983; Wight et al., 1985; Varani et al., 1989). TSP-1 levels in body fluid are very low under normal conditions, but can be transiently elevated under pathological conditions or stimulation by regulatory agents (Lahav et al., 1993; Stern et al., 1996; Yesner et al., 1996). Thus, the regulation of TSP-1 level is important for modulating biological processes and for providing a possible approach to cancer therapy.

The level of TSP-1 protein is controlled by the level of TSP-1 gene transcription, which has been studied 
by several groups. We have demonstrated that upregulation of TSP-1 expression in the human hepatocarcinoma cell line Hep3B by PMA is mediated by increased c-Jun binding to the putative AP-1 site near nucleotide (nt) -1492 (Kim et al., 2001b). However, TSP-1 is negatively regulated by PMA in porcine aortic endothelial (PAE) cells, where leads to c-Juninduced repression mediated by blocking of the binding of an unknown nuclear protein to a site between nt -767 and nt -735 (Kim et al., 2000).

In preliminary experiments, we found that a 5'deletion construct lacking the region upstream of $\mathrm{nt}$ -767 consistently reduced the promoter activity of tsp-1 in PMA-treated or -untreated Hep3B cells, while a further deletion lacking the region upstream of $\mathrm{nt}$ -407 had sharply elevated promoter activity. Cloning of the tsp-1 promoter region has shown that TSP-1 gene expression can be controlled by multiple cisacting elements and trans-activators that influence the transcriptional rate (Hennessy et al., 1989; Laherty et al., 1989). We searched the sequence between $\mathrm{nt}$ -767 and $n t-407$ on the $t s p-1$ promoter and found a consensus sequence for the $Y Y-1$ binding site at the region near nt -440 . The $Y Y-1$ site is found in many genes and displays activating or repressing functions when the $Y Y-1$ factor binds to other trans-acting factors (Weis et al., 1992; Shrivastava et al., 1993; Zhao et al., 1998). The putative YY-1 factor site on the tsp-1 promoter, 5'-tcggcCCATTTTtcttt-3', perfectly resembles the previously sequenced repressive YY-1 site which has a sequence of 5'-CCAT NTT-3' (Shrivastava et al., 1994).

The present study was focused on the YY-1 site to determine the regulatory mechanism of TSP-1 expression in Hep3B cells stimulated with PMA or c-jun cotransfection. The putative $Y Y-1$ site consistently repressed $t s p-1$ promoter activity in the absence of cis-elements in the region between $n t-2,200$ and nt -767 . The inhibitory effect of the potential $Y Y-1$ site was affected by $c-J u n / Y Y-1$ interaction.

\section{Materials and Methods}

\section{Cell line and culture conditions}

The human hepatocarcinoma cell line Hep3B was obtained from the American Type Culture Collection (Rockville, MD). Cells were cultured in RPMI 1640 medium containing $10 \%$ heat-inactivated fetal bovine serum, $100 \mathrm{U} / \mathrm{ml}$ penicillin and $100 \mathrm{U} / \mathrm{ml}$ streptomycin. Cells were maintained at $37^{\circ} \mathrm{C}$ with $5 \% \quad \mathrm{CO}_{2}$. For the following experiments, cells $\left(3 \times 10^{5}\right.$ cells $\left./ \mathrm{ml}\right)$ were seeded on a 6-well plate, cultured for 24 or 48 $\mathrm{h}$, and washed twice with CMF-PBS $(\mathrm{pH} 7.2)$. To evaluate the luciferase activity induced by various deletion constructs, the confluent cells were tran- siently transfected with 2 or $3 \mu \mathrm{g}$ of various plasmids using FuGENE 6 (Boehringer Mannheim, Germany) and washed twice with washing solution after $8 \mathrm{~h}$. To examine the effects of regulatory agents, the washed confluent cells were transferred into fresh serum-free RPMI 1640 medium containing 50 or 100 nM PMA and cultured for the desired duration. Subsequently, culture supernatants were harvested and concentrated to determine the TSP-1 level. To detect YY-1 or c-Jun, the cells were harvested at the indicated times and nuclear extracts were prepared. Cell viability and total protein concentration was checked as in our earlier report (Kim et al., 2001a, b).

\section{Preparations of reporter plasmids containing 5 -deletion constructs of the tsp-1 promoter}

To prepare various reporter plasmids, six fragments derived from genomic tsp-1 DNA were initially prepared by PCR. Three additional 5'-deletion constructs, $\left(3^{\prime}\right),\left(4^{\prime}\right)$ and $\left(5^{\prime}\right)$, were built by the same methods as the initial six fragments. Identical reverse primer was used for each construct, which had the sequence 5'-GATCCTGTAGCAGGAAGCACAAG-3'. The forward primers were 5'-GAGGAAGGGCTTTGTGTTTGAGAT A-3' for fragment (1), 5'-GAAGAGCAAG ATGAATGC TGT-3' for fragment (2), 5'-GAGATGAATGGAATTCC AGGAAG-3' for fragment (3), 5'-GTTTTTCAAGACAC AATTTCA-3' for fragment (3'), 5'-CCCCTTGATGAG AATACGCAC-3' for fragment (4), 5'-TATCCACCTCT CGCCATCAACC-3' for fragment (4'), 5'-ACCGACTTC TCTGAGAAGTTCTA-3' for fragment (5), 5'-CGCCCC TTTAAAAGCGCGCG-3' for fragment (5') and 5'-TCC GGTACACACAGGTAAGT-3' for fragment (6). These forward primers were ligated to a Kpnl-linker at the $5^{\prime}$ end, and the reverse primers had a Xhol-linker at the $5^{\prime}$ end. The products were designated as fragments (1), (2), (3), (3'), (4), (4'), (5), (5') and (6) as shown in Figure 1A. All fragments were gradually shortened by serial deletion from the $5^{\prime}$ end, but have identical $3^{\prime}$ ends (+754). Fragment (1) lacks the sequence upstream of $n t-2,200$, (2) of -1732 , (3) of -1182 , (3') of -860, (4) of $-767,\left(4^{\prime}\right)$ of -407, (5) of $-214,\left(5^{\prime}\right)$ of -35 , and (6) of +138 . Amplification was conducted by $\mathrm{PCR}$ under the following conditions: denaturation at $90^{\circ} \mathrm{C}$ for $30 \mathrm{~s}$, annealing at $50^{\circ} \mathrm{C}$ for $30 \mathrm{~s}$, and extension at $72^{\circ} \mathrm{C}$ for $1 \mathrm{~min}$. The PCR products were cloned into the $\mathrm{Kpnl}$ and $\mathrm{Xhol}$ sites of a promoterless pGL3 luciferase expression vector (Promega, Madison, WI) and termed pTSP-Luc (TSPLuc reporter plasmid), yielding PTSP-Luc-1, -2, -3, -3', $-4,-4^{\prime},-5,-5$ ' and [6 (Figure 1A).

\section{Mutation of the AP-1 or YY-1 sites}

A PCR method using overlap extension, as described by Horton (Horton et al., 1997), was used to convert 


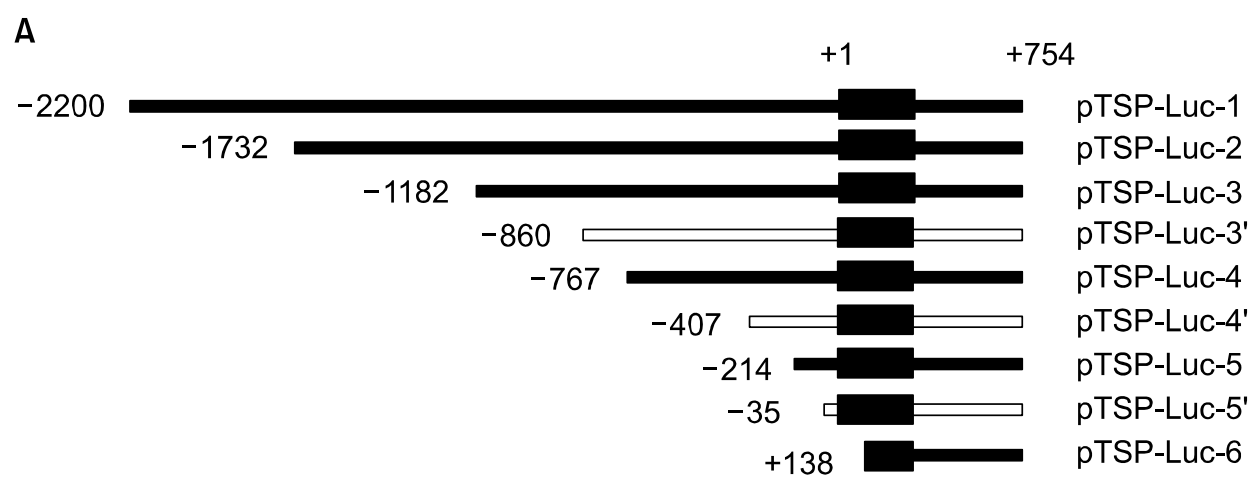

B

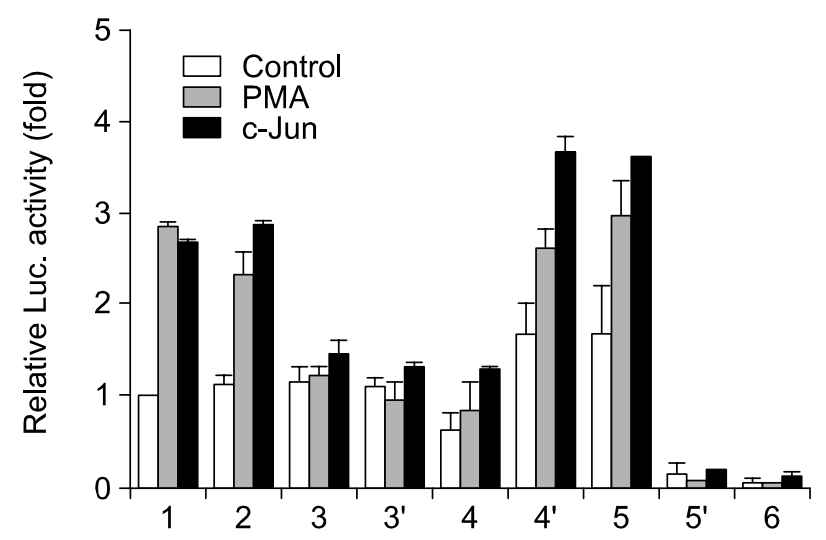

$\begin{array}{llllllllll}\mathrm{AP}-1 & + & + & - & - & - & - & - & - & - \\ \mathrm{YY}-1 & + & + & + & + & + & - & - & - & - \\ \mathrm{GC} & + & + & + & + & + & + & + & - & - \\ \text { CCAAT } & + & + & + & + & + & + & + & - & - \\ \text { TATA } & + & + & + & + & + & + & + & + & - \\ \text { 1st intron } & & & & & & & & & \\ \text { enhancer } & + & + & + & + & + & + & + & + & +\end{array}$

Figure 1. Nine reporter plasmids constructed by serial $5^{\prime}$ deletion $(A)$ and their transcriptional level (B). The six closed bars represent the initial 5 '-deletion constructs and the three open bars represent the secondarily produced constructs created to examine the suppression mechanism of PTSP-Luc-4. Promoter activities mediated by each reporter plasmid are expressed as luciferase level relative to the control level of pTSP-Luc-1, which was normalized against $\beta$-galactosidase activity. It is well known that PMA markedly increases c-Jun and TSP-1 expression in Hep3B cells. Hence, to demonstrate the regulation of tsp-1 promoter activity under highly activated condition, Hep3B cells were stimulated with PMA $(100 \mathrm{nM})$ or c-Jun DNA $(2 \mathrm{mg})$ transfection as strong activators. Relative luciferase levels are expressed as mean \pm SD (bars on the column) from quadruplicate experiments for PMA stimulation or duplicate for c-Jun cotransfection.

$\mathrm{AA} \rightarrow \mathrm{TG}$ at positions -1489 to -1488 for AP-1 mutant, and $A T T \rightarrow G T C$ at positions -440 to -438 for $Y Y-1$ mutant, respectively. Complementary primers encompassing the sequences to be mutated (for AP-1 mutant, 5'-ATTTTATATACATATTGAGTTGCTAGAAAA CAC-3' and 5-GTGTTTTCTAGCAACTCAATATGTAT A TAAAAT-3' and for YY-1 mutant, 5'-GCCCCGTCG GCCCGTCTTTCTTTTTACAAAACG-3' and 5'-CGTTT TGTAAAAAGAAA GACG G G C C GACG G G G C - 3', respectively) were designed. The complementary mu- tagenic primers were used in conjunction with flanking primers (for AP-1 mutant, 5'-ATCTGCAGAGGCATTC CTACAA-3' and 5'-CCTGGAATTCCATTCATCTCTTC G-3', and for YY-1 mutant, 5'-CTC CCCGGGCCCCT TGATGAGAATACGC-3', and 5'-GGATGTCCCGGGCA GCTTTGGTCCTC-3', respectively) in two separate PCR. The first PCR products were $561 \mathrm{bp}$ and 348 bp for AP-1, and $354 \mathrm{bp}$ and $653 \mathrm{bp}$ for YY-1 mutant. These products were purified with a Wizard SV gel and PCR clean-up system (Promega, Medison, USA), 
mixed, and used as a templete in a second PCR using only the flanking primers. This generated a single product of $865 \mathrm{bp}$ for $\mathrm{AP}-1$ and $974 \mathrm{bp}$ for YY-1 mutant, respectively. The PCR product of AP-1 mutant was digested at unique restriction sites $P s t l-E c o R I$, and cloned into Pstl-EcoRI restricted site of pTSP-Luc-1. The PCR product of YY-1 mutant was digested at unique Smal sites, and cloned into Smalrestricted site of PTSP-Luc-1 or PTSP-Luc-3'. PTSPLuc-1 containing mutant $Y Y-1$ site or AP-1 site were designated as $m Y Y-1 / p T S P-L u c-1$ or $m A P-1 / p T S P$ Luc-1. Similarly, pTSP-Luc-3' with mutant YY-1 site was termed $m Y Y-1 / p T S P-L u c-3 '$. Mutants were verified by DNA sequencing analyses.

\section{Transient transfection and the reporter gene assay} Hep3B cells ( 3 or $4 \times 10^{5}$ cells $/ \mathrm{ml}$ ) were plated on a 6 -well culture plate and transiently transfected with 2 or $3 \mu \mathrm{g}$ of various plasmids using FuGENE 6. pTSP. Lucs, pCR 3.1 encoding $\beta$-galactosidase, and the expression vector pSG5 for YY-1 or c-Jun were used in a transfection assay. Eight hours later, the cells were treated with PMA at the desired concentration for $12 \mathrm{~h}$ or were left untreated. Subsequently, total cell lysates were prepared using report lysis buffer (Promega, Medison) and subjected to luciferase and $\beta$-galactosidase assays (Sambrook et al., 2000). Promoter activity is expressed as relative luciferase activity of the control level of PTSP-Luc-1, which was normalized against $\beta$-galactosidase activity. Relative luciferase activity is represented as mean fold of control level of PTSP-Luc-1 (mean \pm SD) in duplicate or quadruplicate experiments. Statistical significance was evaluated using Student's $t$-test (defined as $P \leq 0.05)$.

\section{Hep3B cell supernatant concentration}

Supernatants were concentrated with minor modifications as described previously (Kim et al., 2003). Briefly, Hep3B cells were cultured at an initial density of $1 \times 10^{5} / \mathrm{ml}$. At various time points after the treatment with or without PMA, the media were centrifuged $\left(10,000 \mathrm{rpm}, 20 \mathrm{~min}, 4^{\circ} \mathrm{C}\right)$ and were concentrated up to 5 -fold by using a centricon membrane (Mr 50,000 cutoff, Amicon, Lexington). Final samples were stored at $-70^{\circ} \mathrm{C}$.

\section{Northern blot and Western blot analyses}

For Northern blotting, total cellular RNA was isolated and electrophoresed as described in a previous report (Kim et al., 2001a). The blots were hybridized with digoxigenin-labeled probe and detected by an immunochemical method using a digoxigenin chemiluminescence detection kit (Boehringer Mannheim,
Germany). The probe encoding the sequence of human TSP-1 was prepared as previously described (Kim et al., 2001b). A cDNA probe for human GAPDH was also prepared as a loading control. Western blot analysis was carried out as in our previous report (Kim et al., 2001b) using antibodies against TSP-1 (1:1,000, Neomarkers Inc., CA), YY-1 (1:1,000, Santa Cruz Biotechnology Inc., CA), and c-Jun (1:1,000, Santa Cruz Biotechnology).

\section{Preparation of nuclear extracts and EMSA}

EMSAs were performed with minor modifications as described previously (Joung et al., 2003). Briefly, nuclear extracts were prepared from $50 \mathrm{nM}$ PMAstimulated or unstimulated cells as follows: $3 \times 10^{6}$ cells were washed twice with cold PBS ( $\mathrm{pH} 7.4)$, and collected for $5 \mathrm{~min}$ at $3,000 \mathrm{~g}$ at $4^{\circ} \mathrm{C}$. The pelleted cells were suspended and incubated for $15 \mathrm{~min}$ on ice in $200 \mu$ of ice cold buffer A (10 mM HEPES$\mathrm{KOH}(\mathrm{pH} 7.9), 10 \mathrm{mM} \mathrm{KCl}, 1 \mathrm{mM}$ EDTA, $0.1 \mathrm{mM}$ sodium orthovanadate $10 \mathrm{mM} \mathrm{PMSF}, 1 \mu \mathrm{g} / \mathrm{ml}$ leupeptin, $1 \mu \mathrm{g} / \mathrm{ml}$ aprotinin, and $0.5 \%$ Nonidet $P-40$ ) and centrifuged for $30 \mathrm{~s}$ at $12,000 \mathrm{~g}$ at $4^{\circ} \mathrm{C}$. The pellets were resuspended in $100 \mu$ of ice cold buffer $B(20$ $\mathrm{mM}$ HEPES-KOH (pH 7.9), $420 \mathrm{mM} \mathrm{NaCl}, 1 \mathrm{mM}$ EDTA, $0.1 \mathrm{mM}$ sodium orthovanadate, $1 \mu \mathrm{g} / \mathrm{ml}$ leupeptin, $1 \mu \mathrm{g} / \mathrm{ml}$ aprotinin) and incubated for $60 \mathrm{~min}$ on ice. After centrifugation for $20 \mathrm{~min}$ at $12,000 \mathrm{~g}$ at $4^{\circ} \mathrm{C}$, the supernatant was frozen at $-70^{\circ} \mathrm{C}$ until use. To perform the EMSA, the nuclear extracts $(10 \mu \mathrm{g})$ were incubated with the radiolabeled oligonucleotides, oligo-YY-1. Oligo-YY-1 is a 25 -mer oligonucleotide (5'AGCT-cgtcggcCC ATTTTtctttt-3') bearing a repressive YY-1 sequence from nt -450 to $n t-430$. The complementary oligonucleotides were annealed and endlabeled with a T4 kinase (Promega) using $\left[\gamma^{32} \mathrm{P}\right] \mathrm{dATP}$ (NEN, Boston, USA). For the supershift assay, antiAP-1 $(1: 1,000)$ or anti-YY-1 $(1: 1,000)$ was added to nuclear extracts and incubated for $4 \mathrm{~h}$ at $4^{\circ} \mathrm{C}$ before adding labeled oligo-YY-1. The antibodies used here are polyclonal (Santa Cruz Biotechnology Inc., CA).

\section{Co-immunoprecipitation of YY-1 and C-Jun}

Nuclear extracts were prepared $6 \mathrm{~h}$ after adding PMA (50 nM). Subsequently, they were precleared with protein A-Sepharose (Pharmacia Co., Sweden) for 1 $\mathrm{h}$ at $4^{\circ} \mathrm{C}$ and centrifuged for $30 \mathrm{~s}$ at $12,000 \mathrm{~g}$ at $4^{\circ} \mathrm{C}$ before immunoprecipitation. The supernatants were incubated with protein A-Sepharose-bound antibodies to $Y Y-1 \quad(1: 300)$ or C-Jun $(1: 300)$ in immunoprecipitation buffer containing $20 \mathrm{mM}$ Tris- $\mathrm{HCl}(\mathrm{pH}$ 7.5), $150 \mathrm{mM} \mathrm{NaCl}, 1 \mathrm{mM}$ EDTA, $1 \mathrm{mM} \mathrm{EGTA}, 1$ $\mathrm{mM}$ PMSF, $0.1 \mathrm{mM}$ orthovanadate, $1 \mu \mathrm{g} / \mathrm{ml}$ leupeptin, and $1 \mu \mathrm{g} / \mathrm{ml}$ aprotinin under gentle shaking at $4^{\circ} \mathrm{C}$ overnight. After centrifugation for $30 \mathrm{~s}$ at $12,000 \mathrm{~g}$, 
the immunoprecipitated pellet was washed three times with RIPA buffer containing $50 \mathrm{mM}$ Tris- $\mathrm{HCl}(\mathrm{pH} \mathrm{7.5)}$, $150 \mathrm{mM} \mathrm{NaCl}, 1 \%$ Triton X-100, 0.5\% NP-40, $1 \mathrm{mM}$ EDTA, $1 \mathrm{mM}$ PMSF, $0.1 \mathrm{mM}$ orthovanadate, $1 \mu \mathrm{g} / \mathrm{ml}$ leupeptin, and $1 \mu \mathrm{g} / \mathrm{ml}$ aprotinin. Protein obtained from the pellet was dissolved with $1 \times$ SDS-PAGE sample buffer and electrophoresed on a $10 \%$ SDSPAGE gel. The migrated protein was detected by immunoblotting using anti-YY-1 or anti-c-Jun antiserum at a 1:1,000 dilution.

\section{Results}

\section{Transcriptional activities induced by nine 5 -deletion constructs in PMA-treated or c-Jun-cotransfected Hep3B cells}

We initially constructed six 5'-deletion series to investigate the transcriptional regulation of TSP-1 by putative cis-acting elements on the $t s p-1$ promoter (Figure 1A, closed bars). As shown in Figure $1 \mathrm{~A}$, these constructs were made by serial deletion of the 5 ' end of genomic tsp-1 DNA. The full-length DNA comprises 2,974 bp from $n t-2,220$ to $n t+754$. In the presence of PMA, three of the reporter plasmids, pTSP-Luc-1, -2 and -5 , had markedly elevated luciferase levels (an increase over the unstimulated control level of over 2-fold), whereas the luciferase activity of pTSP-Luc-3 remained at the control level (Figure 1B). The luciferase activity of pTSP-Luc-4 was half that of the control, but the reduction was less pronounced under PMA stimulation or c-jun cotransfection. The extent of the up-regulation of pTSP-Luc-1, -2 , and -5 was especially remarkable in c-jun cotransfected cells. In the absence of PMA, the luciferase level of pTSP-Luc-5 was 1.8-fold higher than that of pTSP-Luc-1. Interestingly, among six constructs, pTSP-Luc-4 always reduced to below half level of that of PTSP-Luc-1, $-2,-3$ and -5 , and independently exerted against PMA (Figure 1B). The transcriptional activity of PTSP-Luc-5, which lacks the region upstream of $n t-214$, was rescued from the suppression induced by the $533 \mathrm{bp}$ from -767 to -215 . The luciferase levels of PTSP-Luc-6, which contains the partial first exon and the intact first intron without the TATA box, were sharply diminished.

To examine whether the down-regulation of pTSP. Luc-4 resulted from the action of a cis-element located between nt -767 and nt -214 , we prepared three additional 5'-deletion constructs around PTSP-Luc-4 and PTSP-Luc-5, and called them pTSP-Luc-3', pTSPLuc-4' and PTSP-Luc-5' (Figure 1A). PTSP-Luc-3' containing $Y Y-1$ site reveals level similar to those of pTSP-Luc-3 and -4 (Figure 1B). Surprisingly, in spite of the further deletion in length from pTSP- Luc-4, pTSP-Luc-4' completely recovered its transcriptional activity to the level equal to that of PTSP-Luc-1, -2, and -5 . The suppressive activity of PTSP-Luc-4 was weakened by the presence of the region upstream of position -767, which contains the AP-1 site, and was inactivated by PMA-stimulation or c-jun cotransfection. These data suggest that the PTSP- Luc-4-induced repressive activity be affected by the AP-1 site and by higher expression of C-Jun. Since deletion of the region between $\mathrm{nt}-767$ and $\mathrm{nt}-407$ led to these changes in activity, the repressive activity seen in pTSP-Luc-4 might be generated by the YY-1 site located at nt -440 . We therefore examined the promoter activities of $m A P-1$ and $m Y Y-1$ and the interactions between $Y Y-1$ factor and $c$-Jun.

\section{Promoter activities affected by site-directed mutagenesis on the AP-1 or YY-1 site}

Primarily, two oligonucleotides bearing mutated $Y Y-1$ sequence, $m Y Y-1 A(A T T \rightarrow$ TAT) and $\mathrm{mYY}-1 \mathrm{~B}(\mathrm{ATT} \rightarrow$ GTC) were made to check their binding abilities with YY-1 factor prior to producing the mutant pTSP-Luc-1 and -3 '. Only $m Y Y-1 B$ could not bind with $Y Y-1$ factor from the nuclear extracts in Figure $2 \mathrm{~A}$. Therefore, to determine whether TSP-1 promoter activity is affected by mutation of $Y Y-1$ site, $Y Y-1$ sites in the pTSPLuc-1 and -3' were changed into GTC instead of ATT and their luciferase levels were evaluated (Figure 2B). pTSP-Luc-1 containing mutant YY-1 (mYY-1/pTSPLuc-1) expressed higher level (over 2-fold) than that of native PTSP-Luc-1 without PMA (Inserted figure in Figure 2B). PMA-stimulated mYY-1/pTSP-Luc-1 increased 1.8-fold to the unstimulated one (Figure 2B). To confirm the effects of PMA on the luciferase activity without AP-1 site, transcriptional levels induced by mutant pTSP-Luc-3' (mYY-1/pTSP-Luc-3') was determined in the absence or presence of PMA and represented as relative fold of control level derived from the unstimulated construct. In the presence of PMA, it upregulated about 2-fold (Figure 2B). On the other hand, the PMA-stimulated mutant pTSP-Luc-1 at AP-1 site (mAP-1/pTSP-Luc-1) exerted similar level in transcription comparing with that of unstimulated one.

\section{Time-dependent synthesis of TSP-1 protein and mRNA after stimulation with PMA}

To confirm the regulation of TSP-1 expression in PMA (50 nM)-treated Hep3B cells, the levels of TSP-1 protein and mRNA were determined by Western and Northern blot analysis, respectively (Figure 3). TSP-1 expression was enhanced in a time-dependent manner; TSP-1 mRNA started to increase at $1 \mathrm{~h}$ and reached a maximal level of four times the control level at $3 \mathrm{~h}$. The increased level was maintained until 6 $\mathrm{h}$ and slowly declined thereafter (Figure 3A). As 
A
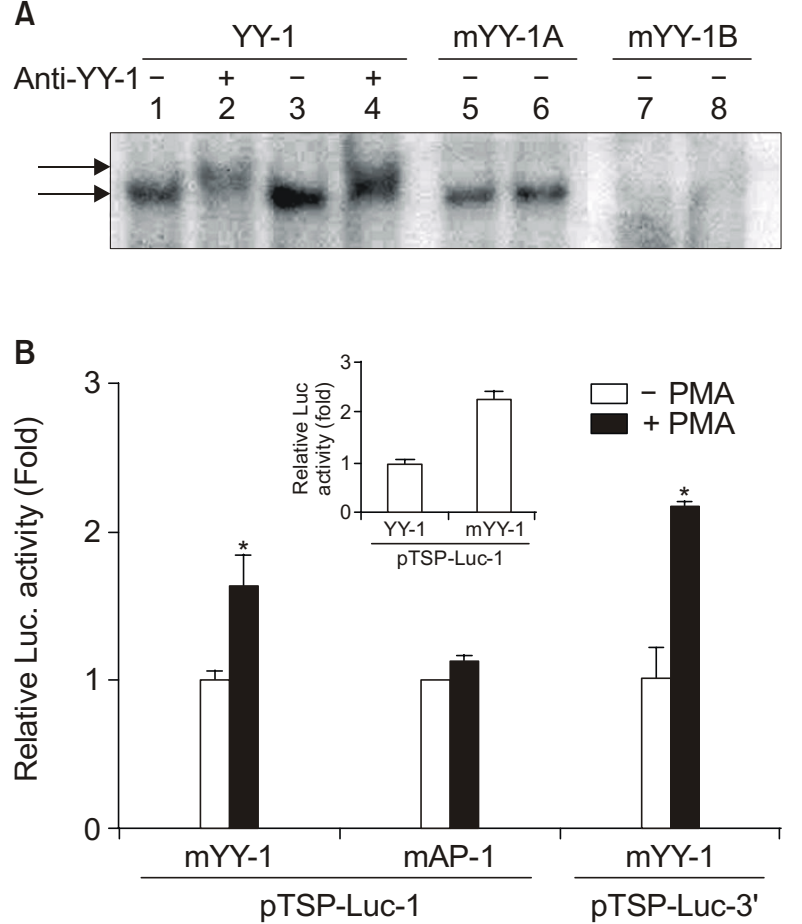

Figure 2. EMSA for synthetic oligonucleotides containing mutant or native YY-1 site $(A)$ and promoter activities of mutants PTSP-Luc-1 and $-3^{\prime}$ at AP-1 or at YY-1 site (B). Two oligonucleotides with mutant YY-1 site were made as follows; ATT was substituted to TAT (mYY-1A) or to GTC (mYY-1B) in the 25-mer oligonucleotide containing the sequence between -451 and -426 on the tsp-1 promoter. For the EMSA nuclear extracts were prepared from cultured Hep3B cells expressing basal level (Lane 1, 2, 5, and 7) or higher level of YY-1 (Lane 3, 4, 6, and 8). Overexpressed cells were generated by $Y Y-1$ gene transfection. For the supershift assay, nuclear extracts incubated with antibody specific to YY-1 for $4 \mathrm{~h}$ $(1: 1000)$ and then mixed with labeled each oligo YY-1. As shown in lane 7 and $8, m Y Y-1 B$ lost binding activity with YY-1 factor. Thus, YY-1 site in the PTSP-Luc-1 and $-3^{\prime}$ were replaced from ATT to GTC for determining luciferase level in Figure 2B. To define the effect of mutant YY-1 site on the transcriptional level in the absence of PMA, transcriptional level of the mutant PTSP-Luc-1 was expressed as relative luciferase level to the native PTSP-Luc-1 level (means $\pm S D$ ) from two separate experiments (the inserted figure in Figure $2 B$ ). Others were represented as relative luciferase level to each level in the unstimulation (mean \pm S.D.) from two separate experiments. *represents statistical significance $(P \leq 0.05)$.

shown in Figure 3B, the level of TSP-1 protein rose in parallel with the level of TSP-1 mRNA, becoming elevated at $6 \mathrm{~h}$ and continuously increasing until 24 h (Figure 3B, upper panel). Elevated levels of protein could be detected at $3 \mathrm{~h}$ in concentrated media (Figure 3B, lower panel). We have previously demonstrated that TSP-1 protein is synthesized at a considerable level until $48 \mathrm{~h}$ (Kim et al., 2001a; Kim
A

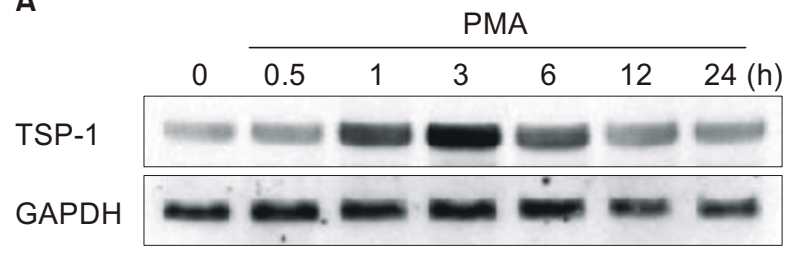

B

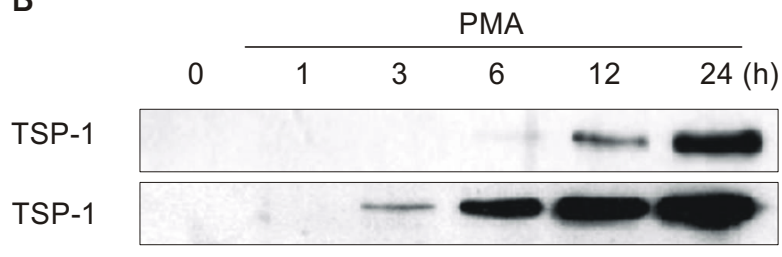

Figure 3. Time-dependent expression of tsp-1 mRNA (A) and protein (B) obtained from the PMA-treated Hep3B cells. Cells $\left(2 \times 10^{5}\right.$ cells $/ \mathrm{ml}$ ) were cultured for $48 \mathrm{~h}$ in RPMI medium containing $10 \%$ heat-inactivated fetal calf serum and then washed twice. The washed cells were cultured for the indicated periods in the serum-free medium. Total cellular RNA $(10 \mu \mathrm{g})$ was used for Northern blot analysis and the migrated bands were visualized by the ECL method using a DIG-labeled TSP-1 probe. Western blot analysis was performed with $100 \mu \mathrm{g} /$ lane of non-concentrated ( $B$, upper panel) or concentrated (B, lower panel) culture supernatants for demonstrating TSP-1 protein level. GAPDH mRNA was used as a control.

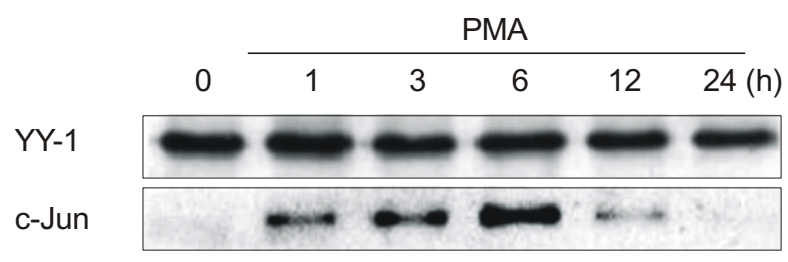

Figure 4. Changes in YY-1 and C-Jun levels in the PMA-treated Hep3B cells. One hundred $\mu \mathrm{g}$ of protein was applied to each lane for Western blot analysis and the migrated bands were visualized using an ECL system as described in the Materials and Methods.

et al., 2003).

\section{Synthesis of YY-1 factor and C-Jun in PMA-stimulated Hep 3B cells}

The YY-1 factor was constitutively synthesized following PMA stimulation and was maintained at constant level until $24 \mathrm{~h}$ (Figure 4 upper panel). In the presence of $50 \mathrm{nM}$ PMA, c-Jun synthesis was up-regulated after $1 \mathrm{~h}$ and reached its maximal level at 6 h (Figure 4, bottom panel). The increased c-Jun level gradually decreased thereafter and returned to the control level at $24 \mathrm{~h}$. 


\section{Binding of nuclear proteins to the putative $Y Y-1$ sequence on the $t s p-1$ promoter}

The remarkable reduction in the luciferase activity of pTSP-Luc-4 may result from the activation of a cis-element within the 360 bp between nt -767 and nt -407 . In order to determine whether the putative YY-1 site within this region is involved in the transcriptional suppression of pTSP-Luc-4, a doublestranded oligonucleotide was prepared for EMSA. It covered the region from -430 to -450 that contains the putative $Y Y-1$ site on the tsp-1 promoter and was designated oligo-YY-1. Nuclear proteins from Hep3B cells bound to oligo-YY-1; the binding level was reduced from $1 \mathrm{~h}$ to $6 \mathrm{~h}$ after PMA stimulation (Figure $5 A$ ), and was dependent on the c-Jun level. The binding was specifically blocked by unlabeled oligo-YY-1 (lane 1 from the left in Figure 5A). In a supershift test, the electrophoretic mobility of oligo$Y Y-1$ was specifically supershifted by an antibody to the $Y Y-1$ factor (Figure 5B), but antibodies against

A

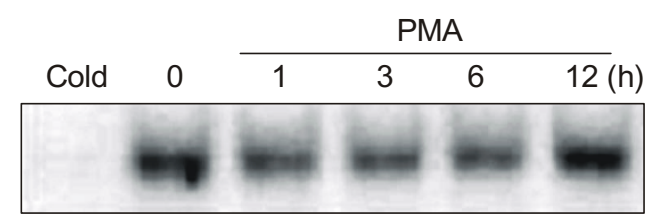

B

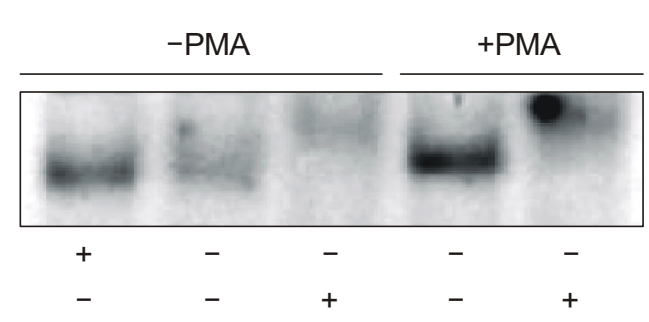

Anti-lgG

Anti-YY-1

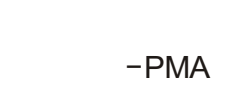

C

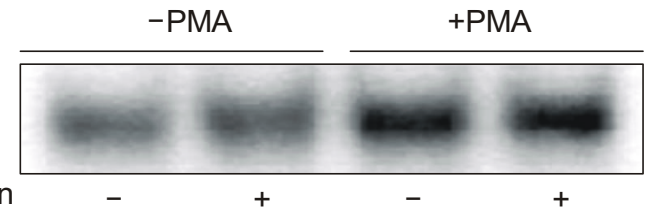

Anti-c-Jun

$+$

Figure 5. EMSA of the nuclear proteins derived from Hep3B cells with labeled double-stranded oligonucleotides containing the putative YY-1 sequence (oligo-YY-1) (A) and supershift patterns using specific antibodies to YY-1 (B) or C-Jun (C). "Cold" in (A) represents unlabeled oligo-YY-1 which was preincubated with stimulated nuclear extracts as a competitor. For the supershift test, nuclear extracts were prepared after adding PMA $(50 \mathrm{nM})$ and incubated with an antibody specific to YY-1 for $4 \mathrm{~h}$ and then mixed with labeled oligo-YY-1. A specific antibody against rabbit $\lg G$ was used as a negative control (lane 1 from the left in Figure 5B). Nuclear protein binding to oligo-YY-1 was reduced from $1 \mathrm{~h}$ until $6 \mathrm{~h}$ after treatment with PMA, and depended on the c-Jun level (Figure 4). The migrated bands were supershifted by the YY-1- specific antibody, but not by antibodies to $\lg G$ or C-Jun.
IgG and c-Jun did not supershift it (Figure 5B and $C)$. These results strongly support that the bound nuclear protein is the $\mathrm{YY}-1$ factor and that c-Jun interferes with its binding to the $Y Y-1$ site.

\section{Formation of a complex of c-Jun with YY-1 factor in nuclear extracts obtained from Hep3B cells}

In the EMSA experiments using oligo-YY-1, the binding level of nuclear protein was reduced depending on c-Jun level. Therefore, c-Jun might regulate the binding of $Y Y-1$ to its responsive site through a protein-protein interaction. To test whether the weakening of the repressive $Y Y-1$ site on the tsp-1 promoter was caused by c-Jun binding to the $Y Y-1$ factor in the nuclear extracts derived from PMAtreated Hep3B cells, nuclear proteins were immunoprecipitated using specific anti-YY-1. The precipitated proteins were dissolved and electrophoresed using SDS-PAGE. The migrated proteins were blotted onto a nitrocellulose membrane and incubated with anti$Y Y-1$ or anti-c-Jun antisera to detect the protein

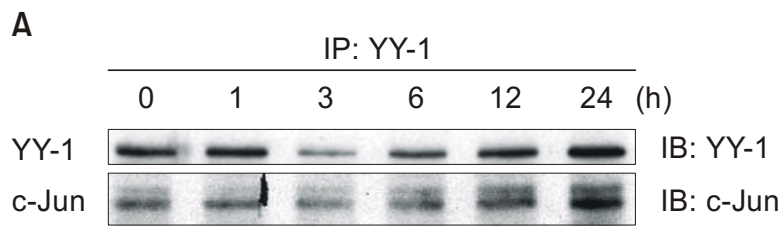

B

\section{Oligo-YY-1}

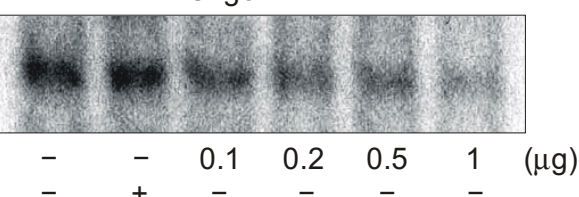

PMA

Figure 6. Co-immunoprecipitation experiments and the effects of exogenous c-Jun. Nuclear proteins obtained from PMA-treated or -untreated Hep3B cells were immunoprecipitated using anti-YY-1 antiserum (A) and the effects of exogenous c-Jun on the binding of nuclear proteins to oligo-YY-1 were tested (B). In order to remove nonspecific precipitated proteins, nuclear extracts were preincubated with protein-A-Sepharose and the supernatants were harvested. The harvested supernatants were immunoprecipitated with a specific YY-1 antibody (1:300) and centrifuged at $13,000 \mathrm{~g}$ for $30 \mathrm{~s}$. After centrifugation, the collected pellet was triple-washed and lysed as described in the Materials and Methods. To identify the nuclear proteins in the lysed precipitates, electrophoresis was carried out for Western blot analysis using a 10\% SDS polyacrylamide gel. The migrated protein bands were detected using an ECL system and antibodies specific to YY-1 or c-Jun. Since the supernatant of each sample was not fully harvested to prevent floating sediments after preincubation, their volume was not identical. Thus, the intensities of the migrated bands do not represent time-dependent changes in protein levels, but only the co-existence of c-Jun and YY-1. 
bands. As shown in Figure $6 \mathrm{~A}$, an antibody to the YY-1 factor co-immunoprecipitated c-Jun with YY-1 factor. To further confirm the interactions between YY-1 and C-Jun, an EMSA test was performed with the addition of exogenous c-Jun (Figure 6B). The binding of $Y Y-1$ to the $Y Y-1$ site decreased by increasing the exogenous c-Jun level.

\section{Discussion}

TSP-1 was known to be a negative regulator in tumorigenesis and angiogenesis. There is considerable interest in its level under normal and pathological conditions. TSP-1 levels in body fluids are normally very low, but are cell-type specifically regulated by various regulatory agents (Bornstein, 1992; Stern et al., 1996; Yesner et al., 1996; Kim et al., 2001a). For example, PMA up-regulates TSP-1 in the human hepatocarcinoma cell line Hep3B (Kim et al., 2001b), but down-regulates it in PAE cells (Kim et al., 2000; Kim et al., 2001a) All-trans retinoic acid exerts the opposite effects against PMA in two type cells (Kim et al., 2001a).

It is generally accepted that TSP-1 levels are tightly regulated at the transcriptional level (Shrivastava et al., 1989; Bornstein et al., 1990; Castle et al., 1993; Framson et al., 1993; Mettouchi et al., 1994; Slack et al., 1994; Tikhonenko et al., 1996; Salnikow et al., 1997; Dejong et al., 1999). However, there is relatively little information on the molecular mechanisms of TSP-1 expression. In preliminary experiments, we observed that 5 '-truncated constructs lacking the region upstream of $n t-767$ had down-regulated TSP-1 transcription. Based on this observation, human genomic TSP-1 DNA (comprising 2,974 bp from $-2,220$ to +754 ) was serially deleted from the $5^{\prime}$ terminus to analyze the detailed molecular mechanisms of its transcription, and six PTSP-Luc reporter plasmids were created (Figure 1A, closed bars). Following stimulation by PMA or C-Jun overexpression, the luciferase level of pTSP-Luc-1 and -2 increased approximately 3 -fold relative to the control level, while the level of pTSP-Luc-3 decreased (Figure 1B). In our previous experiments, transcriptional elevation by PMA or c-jun cotransfection resulted from increased c-Jun binding to the AP-1 site at nt -1492 (Kim et al., 2001b). Thus, the down-regulation of pTSP-Luc-3 might be caused by the missing AP-1 site. Especially, the luciferase activity of pTSP-Luc-4 was always reduced to half of the level of PTSP. Luc-1, $-2,-3$ and -5 in the cells without PMA treatment (Figure 1B). This might be due to the activation of some cis-element between -767 and -214 regions. The down-regulation of PTSP-Luc-4 was less prominent following c-Jun overexpression. Hence, we prepared three additional 5'-truncated constructs, pTSP-Luc-3', 4' and -5', and determined their luciferase levels. Unexpectedly, the transcriptional level of pTSP-Luc-4', which lacks 360 bp within pTSPLuc-4, recovered to that of PTSP-Luc-1. We had previously searched the sequence of the $t s p-1$ promoter to find potential cis-elements in the $360 \mathrm{bp}$ spanning -767 to -407 , and identified a consensus sequence at nt $-440,5$ '-CCATTTT-3', that closely resembles the repressive $Y Y-1$ sequence, 5'CCATNTT-3' (Shrivastava et al., 1994). In the present study, since all cells were cultured with serum-free media after adding the regulatory agents, any effects of SRE (serum response element) and NF-Y were ruled out (Framson et al., 1993). The constant suppression of pTSP-Luc-4, therefore, probably reflects the activity of the putative $Y Y-1$ site, and suggests that TSP-1 expression is suppressed by the putative $Y Y-1$ site when critical cis-elements upstream of the YY-1 site are deleted or mutated.

To further investigate if the suppressive effect of pTSP-Luc-4 on the TSP-1 expression was specifically mediated by $Y Y-1$ site at $n t-440$, we determined luciferase level induced by $\mathrm{mYY}-1$ in the pTSP-Luc-1 and $-3^{\prime}$ (Figure 2B). mYY-1/pTSP-Luc-1 remarkably elevated over 2-fold to the native construct in the absence of PMA. However, it enhanced to higher level in the presence of PMA than its level in the absence of PMA. The elevated level induced by $m Y Y-1 / p T S P-L u c-1$ might be due to the loss of repressive activity expressed by $Y Y-1$ site. $Y Y-1$ factor, which was constitutively expressed at limited level, was not regulated by PMA, but its binding activity was affected by PMA-induced c-Jun. Sequentially, the increased c-Jun could be partially interacted with YY-1 factor at basal level and the residual c-Jun functioned as activator. Hence, mYY-1/pTSP-Luc-1 in the presence of PMA slightly increased transcriptional level compared with its level in the absence of PMA. Nevertheless the pTSP-Luc-3' lacks AP-1 site, mYY-1 of pTSP-Luc-3' responded to PMA and elevated over 2-fold of the unstimulated level (Figure 2B). Four consensus Sp1 binding sites are positioned at nt -71 , $-124,-171$ and -221 , respectively, within the $t s p-1$ promoter. Thus, it seems that overexpressed c-Jun might positively exert transcriptional level through interacting with other factors such as Sp1. Since other upstream cis-elements from nt -440 site regulate promoter activity, mutant PTSP-Luc-3' induced similar level in transcription compared with native one without PMA (data not shown). Moreover, in an EMSA study using oligo-YY-1, nuclear protein binding was decreased by c-Jun overexpression and exogenously added c-Jun. The present data thus reemphasize that the putative $Y Y-1$ represses $t s p-1$ promoter activity, and the repression may be weakened by C-Jun/AP-1 
site interactions. To check whether c-Jun affected the repressive $Y Y-1$ activity, nuclear proteins derived from PMA-treated cells were immunoprecipitated with an antibody specific to the $Y Y-1$ factor and identified by Western blot analysis (Figure 5). C-Jun precipitated together with the $Y Y-1$ factor. This is clear evidence for the direct physical interaction between c-Jun and the $Y Y-1$ factor and the first observation that the weakening of the repressive $Y Y-1$ site is mediated by the formation of a c-Jun/YY-1 factor complex. In an earlier study on the human granulocyte-macrophage colony-stimulation factor gene, the YY-1 factor repressed transactivation mediated by $\mathrm{C}-\mathrm{J}$ un, because of the overlap of the $Y Y-1$ sequence with the AP-1 sequence ( $\mathrm{Ye}$ et al., 1996). Our results (Figure 4A and $5 B$ ) indicate that $\mathrm{C}$-Jun interferes with the binding to the $Y Y-1$ site through the formation of a complex between $c-J u n / Y Y-1$ that cannot bind to the $Y Y-1$ site. Therefore, we firstly showed that c-Jun affects the YY-1 site by forming a complex with YY-1 factor without overlapping between both consensus sequences.

Since the constructs in the 5 '-deletion series without the -440 region, PTSP-Luc-4', $-5,-5$ ', and -6 , are free from the $Y Y-1$ repression, we assumed that their transcriptional level could be recovered. Despite the retention of transcriptional activity of pTSP-Luc-4' and -5 at a high level--- indeed the luciferase levels induced by PTSP-Luc-4' and -5 were higher than those of PTSP-Luc-1 and -2 --- the activity of pTSP-Luc-5' and PTSP-Luc-6 were diminished. The higher level induced by PTSP-Luc-1 or -2 was remarkable in the c-Jun cotransfection. Among the 5 '-truncated series, PTSP-Luc-1 and -2 have almost the full-length $t s p-1$ promoter, and they display a net activity that is achieved through the activation and repression of multiple cis-acting elements in the $t s p-1$ promoter (Laherty et al., 1989; Framson et al., 1993; Salnikow et al., 1997). Since the activity of the $Y Y-1$ factor was diminished by the formation of a $Y Y-1 /$ AP-1 complex, the transcriptional levels of pTSPLuc-1 and -2 might be weakly suppressed by the YY-1 factor under conditions of PMA stimulation or c-Jun overexpression. Therefore, their net transcriptional level is mainly regulated by interactions between the strong transactivator $\mathrm{c}$-Jun and the AP-1 site. TSP-Luc-4' and -5 might be freed from the suppression by the loss of the $Y Y-1$ site. Another possible explanation is that the overexpressed C-Jun interacts with some other transactivators to elevate transcription levels.

In our experiments, PTSP-Luc-3, 3', 4, -4' and -5 induced at a higher level under conditions of c-Jun overexpression than in control conditions, but their extents in the increased level were dependent on the presence of $Y Y-1$ site. Hence, the increased levels stimulated by PTSP-Luc-3, 3' and 4 were lower than those of PTSP-Luc-4' and 5 resulting from co-existence of the inhibitory effects by $Y Y-1$ site and of the stimulatory effects by the remaining free c-Jun/YY-1 complex. On the contrary, because pTSP-Luc-4' and 5 lack $Y Y-1$ site, they remarkably enhanced due to the stimulatory effect of free c-Jun without inhibitory effect. In several reports, c-Jun acts as a superactivator by interacting with Sp1 (Ye et al., 1996; Kardassis et al., 1999). Based on the previous reports, although the suppressive activity induced by YY-1 site affected on the luciferase activities of the pTSP-Luc-3, 3' and 4, free c-Jun might interact with Sp1 and subsequently elevated promoter activity. pTSP-Luc-5' possesses a TATA-box and an enhancer site in the first intron, but PTSP-Luc- 6 contains the first intron enhancer alone. However, pTSP-Luc-5' was produced by deleting the region upstream of $n t$ -35 to remove the effects of cis-elements other than the TATA-box. Because a GC-box-like sequence was identified near the TATA-box, the construct had no extra nucleotides at the $5^{\prime}$ end beyond the consensus sequence of the TATA-box. We therefore cannot exclude that the deficiency in transcription may be caused by insufficient space for basal transcription complex binding. The results indicate that TATA-box is essential for basal transcription of the TSP-1 gene, but that the enhancer in the first intron has no effect, which is in discord with the results of a previous report (Laherty et al., 1989). Our result is supported by an observation from another group, in which the first intron showed no evidence for transcriptional activation (Castle et al., 1993).

In summary, we demonstrate that the repressive effect of YY-1 site at nt -440 on the TSP-1 expression was weakened by other activating sites on the upstream from nt -440 and by PMA via $Y Y-1 / c-J u n$ complex formation.

\section{Acknowledgment}

This work was supported by Korea Research Foundation Grant (KRF-2002-015-EP0033)

\section{References}

Bornstein P, Alfi D, Devarayalu S, Framson P, Li P. Characterization of the mouse thrombospondin gene and evaluation of the role of the first intron in human gene expression. $J$ Biol Chem 1990;265:16691-8

Bornstein P. Thrombospondins: structure and regulation of expression. FASEB J 1992;6:3290-9

Castle VP, Ou X, Rourke KO, Dixit VM. High level thrombospondin 1 expression in two NIH 3 T3 cloned lines confers serum- and anchorage-independent growth. J Biol Chem 1993;268:2899-903 
Chen $\mathrm{H}$, Herndon ME, Lawler J. The cell biology of thrombospondin-1. Matrix Biol 2000;19:597-614

Dejong V, Degeorges A, Filleur S, Ait-Si-Ali S, Mettouchi A, Bornstein $\mathrm{P}$, Binetruy $\mathrm{P}, \mathrm{Cabon} \mathrm{E}$. The Wilms' tumor gene product represses the transcription of thrombospondin 1 in response to overexpression of c-Jun. Oncogene 1999;18: 3143-51

Framson $\mathrm{P}$, Bornstein $\mathrm{P}$. A serum response element and a binding site for NF-Y mediate the serum response of the human thrombospondin 1 gene. J Biol Chem 1993;268: 4989-96

Hennessy SW, Frazier BA, Kim DD, Deckwerth TL, Baumgartel DM, Rotwein P, Frazier WA. Complete thrombospondin mRNA sequence includes potential regulatory sites in the $3^{\prime}$ untranslated region. J Cell Biol 1989;108: 729-36

Horton RM. in vitro recombination and mutagenesis of DNA, A.B. White (Eds.), In: Methods in Molecular Biology, 1997, 141-9. Humana Press Inc., Totowa, NJ

Iruela-Arispe ML, Lombardo M, Krutzsch HC, Lawler J, Roberts DD. Inhibition of angiogenesis by thrombospondin-1 is mediated by 2 independent regions within the type 1 repeats. Circulation 1999;100:1423-31

Jaffe EA, Ruggiero JT, Leung LK, Doyle MJ, McKeownLongo PJ, Mosher DF. Cultured human fibroblasts synthesize and secrete thrombospondin and incorporate it into extracellular matrix. Proc Natl Acad Sci 1983;80:998-1002

Jimenez B, Volpert OV, Crawford SE, Febbraio M, Silverstein $R L$, Bouck N. Signals leading to apoptosis-dependent inhibition of neovascularization by thrombospondin-1. Nat Med 2000;6:41-4

Joung YH, Park JH, Park TK, Lee CS, Kim OH, Ye SK, Yang UM, Lee KJ, Yang YM. Hypoxia activates signal transducers and activators of transcription 5 (STAT5) and increases its binding activity to the GAS element in mammary epithelial cells. Exp Mol Med 2003;5:350-7

Kardassis D, Papakosta P, Pardali K, Moustakas A. C-Jun transactivates the promoter of the human $\mathrm{p} 21^{\text {WAF1/Cip } 1}$ gene by acting as a superactivator of the ubiquitous transcription factor Sp1. J Biol Chem 1999;274:29572-81

Kim SA, Kang JH, Cho IH, Bae SW, and Hong KJ. Cell-type specific regulation of thrombospondin-1 expression and its promoter activity by regulatory agents. Exp Mol Med 2001a; $33: 117-23$

Kim SA, Um SJ, Kang JH, Hong KJ. Expression of thrombospondin-1 in human hepatocarcinoma cell lines and its regulation by transcription factor Jun/AP-1. Mol Cell Biochem 2001b;216:21-6

Kim SA, Hong KJ. Responsive site on the thrombospondin-1 promoter to down-regulation by phorbol 12-myristate in porcine aortic endothelial cell. Exp Mol Med 2003;2:135-40

Lahav J. The functions of thrombospondins and its involvement in physiology and pathophysiology. Biochim Biophys Acta 1993;1182:1-14

Laherty CD, Gierman TM, Dixit VM. Characterization of the promoter region of the human thrombospondin gene. DNA sequences within the first intron increase transcription. J Biol Chem 1989;264:11222-7

Lawler J. The structural and functional properties of thrombospondin. Blood 1986;67:1197-209

Mettouchi A, Cabon F, Montreau N, Vernier P, Mercier G, Blangy $D$, Tricoire $H$, Vigier $P$, Binetruy $B$. SPARC and thrombospondin genes are repressed by the $c$-jun oncogene in rat embryo fibroblasts. EMBO J 1994;13:5668-78

Mosher DF, Doyle ML, Jaffe EA. Synthesis and secretion of thrombospondin by cultured human endothelial cells. J Cell Biol 1982;93:343-8

Nor JE, Mitra RS, Sutorik MM, Mooney DJ, Castle VP, Polverini PJ. Thrombospondin-1 induces endothelial cell apoptosis and inhibits angiogenesis by activating the caspase death pathway. J Vasc Res 2000;37:209-18

Roberts DD. Regulation of tumor growth and metastasis by thrombospondin-1. FASEB J 1996;10:1183-91

Salnikow K, Wang S, Costa M. Induction of activating transcription factor 1 by nickel and its role as a negative regulator of thrombospondin I gene expression, Cancer Res 1997;57:5060-6

Sambrook J, Fritsch EF, Maniatis T, Assay for $\beta$-galactosidase in extracts of mammalian cells. In: J. Sambrook, D.W. Russell (Eds.), Molecular Cloning: A laboratory Mannual 3rd Eds 2000;17:48-51, Cold Spring Harbor, NY

Shrivastava A, Saleque S, Kalpana GV, Artandi S, Goff SP, Calame K. Inhibition of transcriptional regulator Yin-Yang-1 by association with c-Myc. Science 1993;262:1889-92

Shrivastava A, Calame K. An analysis of genes regulated by the multi-functional transcriptional regulator Yin Yang-1. Nucleic Acids Res 1994;22:5151-5

Slack JL, Bornstein P. Transformation by v-src causes transient induction followed by repression of mouse thrombospondin-1. Cell Growth Differ 1994;5:1373-80

Stern M, Savill J, Haslett C. Human monocyte-derived macrophage phagocytosis of senescent eosinophils undergoing apoptosis. Mediation by alpha $v$ beta 3/CD36/thrombospondin recognition mechanism and lack of phlogistic response. Am J Pathol 1996;149:911-21

Streit M, Velasco P, Brown LF, Skobe M, Richard L, Riccardi L, Lawler J, Detmar M. Overexpression of thrombospondin-1 decreases angiogenesis and inhibits the growth of human cutaneous squamous cell carcinomas. Am J Pathol 1999; 155:441-52

Tikhonenko AT, Black DJ, Linial ML. Viral Myc oncoproteins in infected fibroblasts down-modulate thrombospondin-1, a possible tumor suppressor gene. J Biol Chem 1996;271: 30741-7

Varani J, Riser BL, Hughes LA, Carey TE, Fligiel SE, Dixit VM. Characterization of thrombospondin synthesis, secretion and cell surface expression by human tumor cells. Clin Exp Metastasis 1989;7:265-76

Weinstat-Saslow DL, Zabrenetzky VS, VanHoutte K, Frazier WA, Roberts DD, Steeg PS. Transfection of thrombospondin 1 complementary DNA into a human breast carcinoma cell line reduces primary tumor growth, metastatic potential, and 
angiogenesis. Cancer Res 1994;54:6504-11

Weis $L$ and Reinberg D. Transcription by RNA polymerase II: initiator-directed formation of transcription-competent complexes. FASEB J 1992;6:3300-9

Wight TM, Rougi GL, Mumby SM, Bornstein P. Light microscopy immunolocalization of thrombospondin in human tissues, J Histochem Cytochem 1985;33:295-302

Ye J, Zhang X, Dong Z. Characterization of the human granulocyte-macrophage colony-stimulating factor gene promoter: an AP1 complex and an Sp1-related complex trans- activate the promoter activity that is suppressed by an YY1 complex. Mol Cell Biol 1996;16:157-67

Yesner LM, Huh HY, Pearce SF, Silverstein RL. Regulation of monocyte CD36 and thrombospondin-1 expression by soluble mediators. Arterioscler Thromb Vasc Biol 1996;16: 1019-25

Zhao JH, Inoue T, Shoji W. Nemoto $Y$, Obinata M. Direct association of $\mathrm{YY}-1$ with $\mathrm{C}-\mathrm{Myc}$ and the E-box binding protein in regulation of glycophorin gene expression. Oncogene 1998;17:1009-17 